\title{
Path-integral mean-field description of the vortex state in the BEC-to-BCS crossover
}

\author{
J. Tempere, M. Wouters, and J. T. Devreese \\ TFVS, Departement Fysica, Universiteit Antwerpen, Universiteitsplein 1, B2610 Antwerpen, Belgium
}

(Received 11 October 2004; published 30 March 2005)

\begin{abstract}
We derive a path-integral description of the vortex state of a fermionic superfluid in the crossover region between the molecular condensate (BEC) regime and the Cooper pairing (BCS) regime. This path-integral formalism, supplemented by a suitable choice for the saddle point value of the pairing field in the presence of a vortex, offers a unified description that encompasses both the BEC and BCS limits. The vortex core size is studied as a function of the tunable interaction strength between the fermionic atoms. We find that in the BEC regime, the core size is determined by the molecular healing length, whereas in the BCS regime, the core size is proportional only to the Fermi wavelength. The observation of such quantized vortices in dilute Fermi gases would provide an unambiguous proof of the realization of superfluidity in these gases.
\end{abstract}

DOI: 10.1103/PhysRevA.71.033631

PACS number(s): 03.75.Ss, 03.75.Kk, 03.75.Lm

\section{INTRODUCTION}

The realization of superfluidity in dilute fermionic gases [1] has opened up a new avenue for the investigation of fermionic quantum systems. In such gases, the presence of Feshbach resonances in the interatomic scattering allows to tune the interaction strength from strongly repulsive to strongly attractive [2]. In the limit of weak repulsive interactions, deeply bound molecular bosons are formed that Bose condense [1] (BEC). In the limit of weak attractive interactions, a Bardeen-Cooper-Schrieffer (BCS) fermionic superfluid arises [3]. The crossover between both regimes has recently attracted a great deal of theoretical and experimental interest.

One of the hallmarks of superfluidity, be it bosonic or fermionic, is the presence of quantized vortices. Whereas vortices are well understood both in the BEC and the BCS limits, it is not clear how the characteristics of the vortex (such as the core size) behave in the crossover regime. Bulgac and Yu have extended density functional theory to superfluid fermion systems [4], and studied vortex states in the BCS regime within their superfluid local density approximation (SLDA) [5]. They found that in the BCS regime vortices give rise to a depletion in the fermion density. These results confirm earlier calculations based on the Bogoliubov-de Gennes theory [6].

The goal of this paper is to develop a path-integral treatment suited to describe vortices in the BCS, BEC, and crossover regimes. With this treatment, we investigate how the vortex core size and the fermionic density depletion at the core change when the fermionic superfluid is brought from the BEC to the BCS regime. On the BCS side of the Feshbach resonance, we compare our results to those obtained with the SLDA treatment [5].

A path-integral treatment for the ground state of the fermionic superfluid was developed by Sá de Melo, Randeria, and Engelbrecht $[7,8]$. Their formalism provides a unified description of the BEC, BCS, and crossover regimes and predicts a smooth crossover for the critical temperature, pairing gap, and chemical potential. They consider a homogeneous Fermi gas of atoms determined by the action functional

$$
\begin{aligned}
\mathcal{S}_{1}= & \int_{0}^{\beta} d \tau \int d \mathbf{x} \sum_{\sigma}\left[\bar{\psi}_{\mathbf{x}, \tau, \sigma}\left(\frac{\partial}{\partial \tau}-\frac{1}{2 m} \nabla_{\mathbf{x}}^{2}-\mu\right) \psi_{\mathbf{x}, \tau, \sigma}\right] \\
& +\int_{0}^{\beta} d \tau \int d \mathbf{x} g \bar{\psi}_{\mathbf{x}, \tau, \uparrow} \bar{\psi}_{\mathbf{x}, \tau, \downarrow} \psi_{\mathbf{x}, \tau, \downarrow} \psi_{\mathbf{x}, \tau, \uparrow} .
\end{aligned}
$$

In this expression $\bar{\psi}_{\mathbf{x}, \tau, \sigma}$ and $\psi_{\mathbf{x}, \tau, \sigma}$ are the Grassmann variables describing the fermionic degrees of freedom, where $\mathbf{x}$ is the position vector, $\tau$ the imaginary time, and $\sigma=\uparrow, \downarrow$ denotes the two hyperfine spin states present in the Fermi gas. The chemical potential is denoted by $\mu$, and $\beta=1 /\left(k_{B} T\right)$ is the inverse temperature. The interaction between the fermionic atoms only takes place between atoms in different hyperfine spin states, and is described by a contact interaction characterized by the renormalized strength $g$. The partition sum is given by the functional integral over the Grassmann variables

$$
\mathcal{Z}=\int \mathcal{D}\left[\bar{\psi}_{\mathbf{x}, \tau, \sigma}, \psi_{\mathbf{x}, \tau, \sigma}\right] \exp \left\{-\mathcal{S}_{1}\right\}
$$

To unravel the product of four Grassmann variables, the Hubbard-Stratonovic transformation is performed. This transformation introduces the bosonic Hubbard-Stratonovic fields $\Delta_{\mathbf{x}, \tau}$ and $\bar{\Delta}_{\mathbf{x}, \tau}$ such that

$$
\mathcal{Z}=\int \mathcal{D}\left[\bar{\Delta}_{\mathbf{x}, \tau}, \Delta_{\mathbf{x}, \tau}\right] \int \mathcal{D}\left[\bar{\psi}_{\mathbf{x}, \tau, \sigma}, \psi_{\mathbf{x}, \tau, \sigma}\right] \exp \left\{-\mathcal{S}_{2}\right\}
$$

with

$$
\begin{aligned}
\mathcal{S}_{2}= & \int_{0}^{\beta} d \tau \int d \mathbf{x}\left\{\sum_{\sigma}\left[\bar{\psi}_{\mathbf{x}, \tau, \sigma}\left(\frac{\partial}{\partial \tau}-\frac{1}{2 m} \nabla_{\mathbf{x}}^{2}-\mu\right) \psi_{\mathbf{x}, \tau, \sigma}\right]\right. \\
& \left.-\bar{\Delta}_{\mathbf{x}, \tau} \psi_{\mathbf{x}, \tau, \downarrow} \psi_{\mathbf{x}, \tau \uparrow}-\Delta_{\mathbf{x}, \tau} \bar{\psi}_{\mathbf{x}, \tau, \uparrow} \bar{\psi}_{\mathbf{x}, \tau, \downarrow}-\frac{\bar{\Delta}_{\mathbf{x}, \tau} \Delta_{\mathbf{x}, \tau}}{g}\right\} .
\end{aligned}
$$

The action functional $\mathcal{S}_{2}$ is quadratic in the Grassmann variables so that the functional integration over these variables can in principle be evaluated. 


\section{SADDLE POINT FOR THE VORTEX STATE}

In practice, to perform the functional integration over Grassmann variables, one has to choose a saddle point value for the fields $\bar{\Delta}_{\mathbf{x}, \tau}, \Delta_{\mathbf{x}, \tau}$. To describe the ground state, Sá de Melo et al. [7] suitably chose a uniform constant saddle point $\left(\bar{\Delta}_{\mathbf{x}, \tau}\right)^{*}=\Delta_{\mathbf{x}, \tau}=|\Delta|$. After doing this, the Grassmann variables can be integrated out straightforwardly, resulting in an effective saddle-point action

$$
\mathcal{S}_{\text {eff }}=\int_{0}^{\beta} d \tau \int d \mathbf{x}\left\{-\operatorname{tr}\left[\ln \left(-G^{-1}\right)\right]-\frac{|\Delta|^{2}}{g}\right\},
$$

where $\mathbb{G}^{-1}$ is the inverse Nambu propagator, for the ground state

$$
-\mathbb{G}_{\text {ground state }}^{-1}=\sigma_{0} \frac{\partial}{\partial \tau}-\sigma_{1}|\Delta|-\sigma_{3}\left[\frac{1}{2 m} \nabla_{\mathbf{x}}^{2}+\mu\right],
$$

and the $\sigma_{j}$ are Pauli matrices. The saddle point equation $\delta \mathcal{S}_{\text {eff }} / \delta|\Delta|=0$ then leads to the familiar gap equation for the fermionic superfluid [9]. The chemical potential $\mu$ is fixed by the fermion density. At finite temperatures, fluctuations around the saddle point value can be taken into account perturbatively to improve the theory and find the critical temperature $[7,8]$. Alternatively, the finite temperature regime can be studied by introducing a temperature-dependent effective action formalism for the phase field [10], or by variational perturbation theory [11].

To investigate the vortex state, we propose to use a different saddle point, and set

$$
\left(\bar{\Delta}_{\mathbf{x}, \tau}\right)^{*}=\Delta_{\mathbf{x}, \tau}=\left|\Delta_{r}\right| \exp (i \theta)
$$

where $\theta$ is the angle around the vortex line and $r$ is the distance to the vortex line. That is, $r$ and $\theta$ are the radial and angular coordinates if one chooses cylindrical coordinates $\mathbf{x}=(r, \theta, z)$ such that the $z$ axis lies along the (straight) vortex line. This particular choice of the saddle point value lies at the core of the present treatment. With this choice for the saddle point value, the integration over Grassmann variables leads again to an effective action of the form (5), but with a different result for $\mathbb{G}^{-1}$. We find

$$
\begin{aligned}
-\mathrm{G}_{\text {vortex }}^{-1}= & \sigma_{0}\left[\frac{\partial}{\partial \tau}-\frac{i}{2 m r} \mathbf{e}_{\phi} \cdot \nabla_{\mathbf{x}}\right]-\sigma_{1}\left|\Delta_{r}\right| \\
& +\sigma_{3}\left[-\frac{1}{8 m r^{2}}-\frac{1}{2 m} \nabla_{\mathbf{x}}^{2}-\mu\right] .
\end{aligned}
$$

The density of paired atoms near the vortex core $\left(\left|\Delta_{r}\right|\right.$ as a function of $r$ ) can in principle be derived from the saddlepoint equation $\delta \mathcal{S}_{\text {eff }} / \delta\left|\Delta_{r}\right|=0$ (although in practice the presence of the spatial derivatives inhibits straightforward calculation). However, the result no longer contains explicit information on the density of fermionic atoms. The results of Bulgac et al. interestingly show that whereas $\left|\Delta_{r}\right|$ tends to zero at the vortex core, the fermionic density needs not go to zero. To study the density of fermionic atoms, expression (8) is not suited.

\section{EXPLICITLY DETAILING THE FERMIONIC DENSITY}

In order to introduce the fermionic atom density in the path-integral expressions, a transformation was proposed by De Palo et al. [12] based on the identity

$$
\begin{aligned}
C= & \int \mathcal{D}\left[\rho_{\mathbf{x}, \tau}^{H S}, \rho_{\mathbf{x}, \tau}\right] \exp \left[-\int_{0}^{\beta} d \tau \int d \mathbf{x} i \rho_{\mathbf{x}, \tau}^{H S}\right. \\
& \left.\times\left(\rho_{\mathbf{x}, \tau}-\bar{\psi}_{\mathbf{x}, \tau, \uparrow} \psi_{\mathbf{x}, \tau, \uparrow}-\bar{\psi}_{\mathbf{x}, \tau, \downarrow} \psi_{\mathbf{x}, \tau, \downarrow}\right)\right],
\end{aligned}
$$

where $C$ is a constant $c$ number and $\rho_{\mathbf{x}, \tau}^{H S}, \rho_{\mathbf{x}, \tau}$ are bosonic fields. This generalized delta-function expression identifies $\rho_{\mathbf{x}, \tau}$ with $\Sigma_{\sigma} \bar{\psi}_{\mathbf{x}, \tau, \sigma} \psi_{\mathbf{x}, \tau, \sigma}$, the fermionic density.

Multiplying the partition sum (4) with the constant (10), and approximating the Hubbard-Stratonovich fields $\bar{\Delta}_{\mathbf{x}, \tau}, \Delta_{\mathbf{x}, \tau}$ by the vortex saddle point (7) leads to the following saddlepoint approximation for the partition sum:

$$
\mathcal{Z}_{\mathrm{sp}}=\int \mathcal{D}\left[\bar{\psi}_{\mathbf{x}, \tau, \sigma}, \psi_{\mathbf{x}, \tau, \sigma}\right] \int \mathcal{D}\left[\rho_{\mathbf{x}, \tau}^{H S}, \rho_{\mathbf{x}, \tau}\right] \exp \left\{-\mathcal{S}_{\mathrm{sp}}\right\}
$$

with

$$
\begin{aligned}
\mathcal{S}_{\mathrm{sp}}= & \int_{0}^{\beta} d \tau \int d \mathbf{x}\left\{\sum _ { \sigma } \left[\overline { \psi } _ { \mathbf { x } , \tau , \sigma } \left(\frac{\partial}{\partial \tau}+\frac{1}{8 m r^{2}}-\frac{1}{2 m} \nabla_{\mathbf{x}}^{2}-\mu\right.\right.\right. \\
& \left.\left.-i \rho_{\mathbf{x}, \tau}^{H S}\right) \psi_{\mathbf{x}, \tau, \sigma}\right]+i \rho_{\mathbf{x}, \tau}^{H S} \rho_{\mathbf{x}, \tau}-\left|\Delta_{r}\right|\left(\psi_{\mathbf{x}, \tau, \downarrow} \psi_{\mathbf{x}, \tau, \uparrow}\right. \\
& \left.\left.+\bar{\psi}_{\mathbf{x}, \tau, \uparrow} \bar{\psi}_{\mathbf{x}, \tau, \downarrow}\right)-\frac{\left|\Delta_{r}\right|^{2}}{g}\right\} .
\end{aligned}
$$

Note that a term proportional to $\bar{\psi}_{\mathbf{x}, \tau, \sigma} \mathbf{e}_{\phi} \cdot \nabla_{\mathbf{x}} \psi_{\mathbf{x}, \tau, \sigma}$ has been neglected in accordance with Ref. [12], who claim that this term is irrelevant for the low-energy properties. To get rid of the path integration over $\rho_{\mathbf{x}, \tau}^{H S}, \rho_{\mathbf{x}, \tau}$ in Eq. (11), we again use a saddle-point approach and set these fields equal to $\rho_{\mathbf{x}, \tau}^{H S}=\rho_{r}^{H S}$, $\rho_{\mathbf{x}, \tau}=\rho_{r}$. This implies that the fermion density only depends on the distance $r$ from the vortex core (and not on the $\theta$ or $z$ coordinate). From Eq. (12) it is clear that the auxiliary field $i \rho_{r}^{H S}$ is related to the chemical potential. It is useful to introduce [12]

$$
\zeta_{r}=i \rho_{r}^{H S}+\mu-\frac{1}{8 m r^{2}}
$$

On the level of the fermionic degrees of freedom, $\zeta_{r}$ acts as a one-body potential combining the auxiliary field, the chemical potential and the angular momentum barrier. Alternatively, it can be interpreted as a local chemical potential.

To perform the integration over the Grassmann variables we assume that $\zeta_{r}$ and the pairing field $\Delta_{r}$ vary slowly in comparison with the relevant fermion frequencies. After integration over $\bar{\psi}_{\mathbf{x}, \tau, \sigma}, \psi_{\mathbf{x}, \tau, \sigma}$, we then obtain the result $\mathcal{Z}_{\mathrm{sp}}$ $\propto \exp \left\{\mathcal{S}_{\text {eff }}^{\prime}\right\}$ with 


$$
\begin{aligned}
\mathcal{S}_{\mathrm{eff}}^{\prime}= & -\left\{\frac{\left|\Delta_{r}\right|^{2}}{g}-\left(\zeta_{r}-\mu+\frac{\hbar^{2}}{8 m r^{2}}\right) \rho_{r}\right. \\
& \left.+2 \int \frac{d \mathbf{k}}{(2 \pi)^{3}} \ln \left[2 \cosh \left(\frac{\beta}{2} \sqrt{\left(\frac{k^{2}}{2 m}-\zeta_{r}\right)^{2}+\left|\Delta_{r}\right|^{2}}\right)\right]\right\} .
\end{aligned}
$$

\section{PROPERTIES OF THE VORTEX CORE}

The first saddle point equation is

$$
\frac{\partial \mathcal{S}_{\mathrm{eff}}^{\prime}}{\partial \rho_{r}}=0 \Leftrightarrow \zeta_{r}=\mu-\frac{1}{8 m r^{2}} .
$$

This locks the local chemical potential $\zeta_{r}$ as a function of the overall chemical potential and the energy barrier of the vortex flow, as one would expect in the Thomas-Fermi approximation in the molecular limit. The parameter $\mu$ is determined by fixing the total number of particles (or the density far away from the vortex core). Differentiating $\mathcal{S}_{\text {eff }}^{\prime}$ with respect to $\left|\Delta_{r}\right|$ we find the familiar gap equation, but with a local chemical potential determined by $\zeta_{r}$ :

$$
\frac{1}{k_{F} a_{s}}=-\frac{2}{\pi} \int d k k^{2}\left(\frac{\tanh \left(\beta E_{k} / 2\right)}{E_{k}}-\frac{1}{k^{2}}\right),
$$

with $E_{k}=\sqrt{\left(k^{2}-\zeta_{r}\right)^{2}+\Delta_{r}^{2}}$. To obtain this expression, we have used an effective contact interaction potential between the atoms, i.e., the two-body potential is $V(\mathbf{r})=4 \pi \hbar^{2} a_{s} / m \delta(\mathbf{r})$, where $a_{s}$ is the $s$-wave scattering length. The relation between the scattering length and the renormalized interaction strength $g$ is given by [7]

$$
\frac{\pi m}{2 \hbar^{2} a_{s}}=\frac{1}{g}+\int \frac{d \mathbf{k}}{(2 \pi)^{3}} \frac{1}{(\hbar k)^{2} / m} .
$$

Finally, the third saddle point equation $\partial \mathcal{S}_{\text {eff }}^{\prime} / \partial \zeta_{r}=0$ allows to calculate the fermionic density near the vortex core

$$
\rho_{r}=\frac{3}{2} \int d k k^{2}\left[1-\frac{k^{2}-\zeta_{r}}{E_{k}} \tanh \left(\beta E_{k} / 2\right)\right] .
$$

In Eqs. (16) and (18), units are such that wave numbers are expressed in Fermi wave numbers and energies in Fermi energies.

We investigate the solutions of these equations in the zero temperature limit $(\beta \rightarrow \infty)$, so that we may assume that fluctuation corrections around the saddle point values are not important. The result for the pair density and the fermion density are shown in Fig. 1. Both the pair density and the fermion density go to zero in a region with spacial dimensions of the order of $1 / k_{F}$ around the vortex line $(r=0)$. In the BEC limit, the pair density and the fermionic atom density become zero at the same distance from the vortex line, so that no atoms are seen inside the core. This corresponds to the expectation for a molecular BEC. In the BCS limit, fermionic atoms can penetrate into the region where the pair density is zero. These results qualitatively agree with those of Bulgac et al. [5] in the BCS region, but quantitatively they

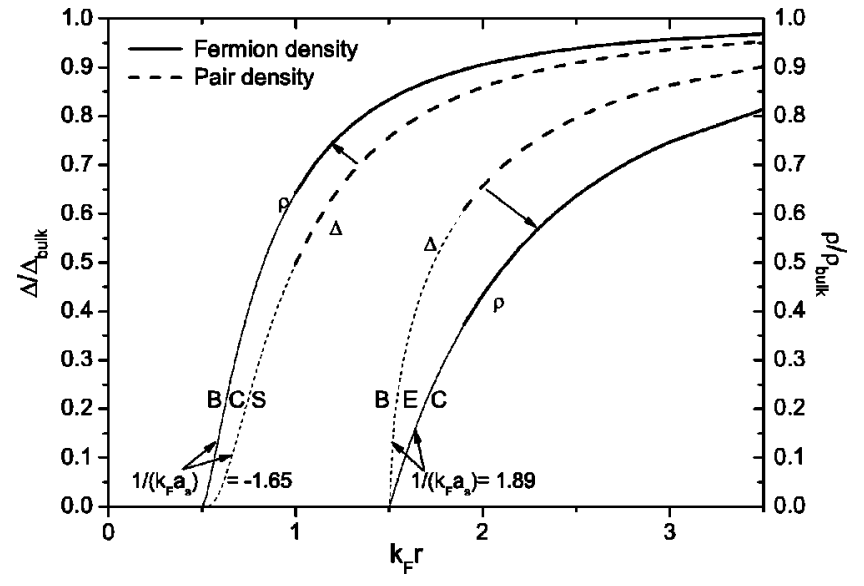

FIG. 1. The pair density relative to the bulk pair density (left axis, dashed curves) and the fermionic atom density relative to its bulk value (right axis, full curves) are shown as a function of the distance to the vortex line, for two different values of the interaction strength. The depletion of the fermion density at the core is overestimated by the current approach, as discussed in the text. The thin part of the curves indicate the region where the current approach is unreliable.

are quite different: in Refs. [5,6] only a small depression in the fermionic density is found on the BCS side of the Feshbach resonance. What can be the reason for the discrepancy? Most likely the assumption that the pairing field $\Delta_{r}$ is smooth on the length scale of the relevant fermion frequencies is too crude. As in the case of the Thomas-Fermi approximation, which breaks down in the region where the order parameter becomes zero, we expect that the assumption that $\Delta_{r}$ varies smoothly breaks down in a region near the point where $\Delta_{r}$ becomes zero. In Fig. 1, the results deemed unreliable are shown in thin curves, whereas the results in the region where the aforementioned assumption is estimated to hold are shown in thick curves. The criterion that we used in Fig. 1 is that if $\boldsymbol{\nabla} \cdot \Delta_{r}>\Delta_{\text {bulk }}\left(\right.$ or $\boldsymbol{\nabla} \cdot \rho_{r}>\rho_{\text {bulk }}$ ) then the pairing field (or fermion density) is no longer smoothly varying and the Thomas-Fermi approximation becomes unreliable. Here dimensionless units based on the Fermi energy and Fermi wave vector are used.

We note moreover that in superfluid neutron matter the semiclassical approximation leads to a spatial profile of the vortex that is similar to the spatial profile obtained in the present treatment [13]. This peculiar spatial profile is an artifact of the semiclassical approximation, and in the weakcoupling limit where the Ginzburg-Landau approach is valid, the solution of the Ginzburg-Landau equations for vortices in a neutron superfluid shows a smooth spatial profile $[13,14]$.

Thus, we must conclude that this assumption may bring us qualitative insight into the core region, but that the quantitative analysis of how exactly $\Delta_{r}$ and $\rho_{r}$ depend on $r$ near the vortex core is misleading. It is not yet clear whether the current result obtained by the saddle-point approximation can be retrieved in the framework of a Bogoliubov-de Gennes theory where similar assumptions (i.e., that $\Delta_{r}$ varies smoothly on the length scale corresponding to the relevant fermion frequencies) are made.

Still, the present results allow us to extract a value for the core size, since for this purpose one does not need to know 


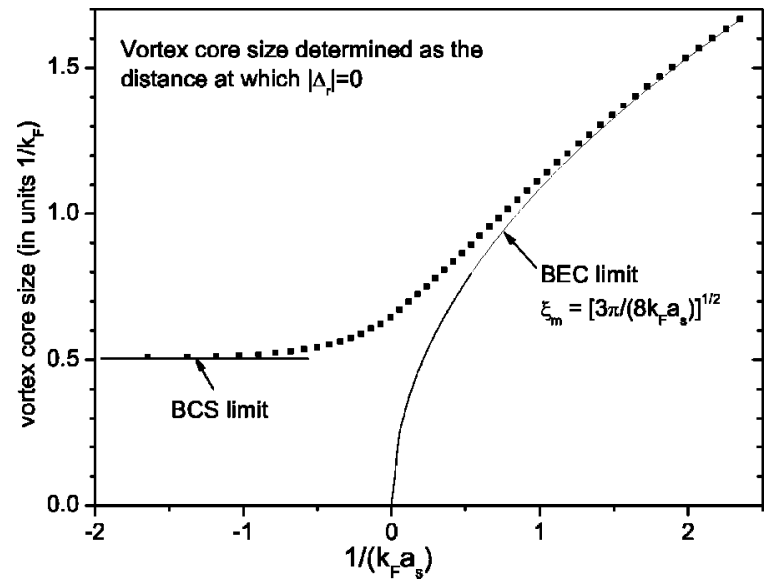

FIG. 2. The vortex core size, in units of $1 / k_{F}$, is shown as a function of the interaction strength $1 /\left(k_{F} a_{S}\right)$. In the BEC regime, the curve shows the molecular healing length, whereas in the BCS limit the result goes to a constant as indicated by the line.

the exact functional dependence of $\Delta_{r}$ on $r$. We estimate the size of the vortex core as the distance from the core at which our solution for $\Delta_{r}$ becomes zero. The result is shown in Fig. 2. In the molecular BEC limit, one expects that the core size is related to the healing length for the molecular BoseEinstein condensate, $\xi_{m}=1 / \sqrt{8 \pi n_{m} a_{m}}$. In the path-integral treatment to lowest order in the fluctuations around the saddle point [8], the molecular scattering length is twice the atomic scattering length $a_{m}=2 a_{s}$. The density of molecules is half the density of atoms if all atoms form molecules $n_{m}$ $=k_{F}^{3} /\left(6 \pi^{2}\right)$. Thus $\xi_{m}=\sqrt{3 \pi /\left(8 k_{F} a_{s}\right)}$ if we express $\xi_{m}$ in units of $k_{F}^{-1}$. Surprisingly, in the BEC limit we find that the vortex core size derived with the path-integral method follows the molecular length $\xi_{m}$ exactly. Approaching the crossover region, the vortex core size deviates from its molecular value. In the BCS limit, the vortex core size tends to a constant, given by $1 /\left(2 k_{F}\right)$, as indicated in Fig. 2 . This suggests that for vortices in ultracold dilute Fermi gases the vortex cores in the BCS limit can be much smaller than the BCS correlation length $\xi_{\mathrm{BCS}}$ (since $k_{F} \xi_{\mathrm{BCS}} \sim E_{F} / \Delta \gg 1$ ), as is the case superfluid vortices in neutron stars [15].

\section{CONCLUSIONS}

The path-integral formalism allows to investigate the ground state of the fermionic superfluid both in the BCS, $\mathrm{BEC}$ and crossover regimes in a unified manner, retrieving the correct limiting behavior $[7,8]$. In this paper we have extended the path-integral treatment to investigate the vortex state of a fermionic superfluid, by introducing an appropriate saddle point for the pairing field. To set up and solve the resulting saddle point equations, the assumption was made that the pairing field varies slowly on the scale of the relevant fermion frequencies. Limitations of this assumption become apparent as one tries to calculate the exact density profile across the vortex core. Nevertheless, with this assumption the vortex core size can be calculated as a function of $1 /\left(k_{F} a_{s}\right)$. The vortex core size varies in the BEC limit according to the molecular BEC healing length, supporting the formalism. The path-integral formalism furthermore predicts that the vortex core size (in units of $1 / k_{F}$ ) tends to a constant value as $1 /\left(k_{F} a_{s}\right)$ is tuned into the BCS regime.

\section{ACKNOWLEDGMENTS}

Insightful discussions with A. Pelster are gratefully acknowledged. The authors also acknowledge discussions with F. Brosens en P. Navez. Financial support for J.T. and M.W. was provided by the Fonds voor Wetenschappelijk Onderzoek-Vlaanderen. This research has been supported financially by the FWO-V projects Nos. G.0435.03, G.0306.00, the W.O.G. project WO.025.99N, the GOA BOF UA 2000 UA. J.T. gratefully acknowledges support of the Special Research Fund of the University of Antwerp, BOF NOI UA 2004.
[1] M. Greiner et al., Nature (London) 426, 537 (2003); M. W. Zwierlein et al., Phys. Rev. Lett. 91, 250401 (2003); S. Jochim et al., Science 302, 2101 (2003).

[2] M. Bartenstein et al., Phys. Rev. Lett. 92, 120401 (2004); T. Bourdel et al., ibid. 93, 050401 (2004).

[3] C. A. Regal et al., Phys. Rev. Lett. 92, 040403 (2004); M. W. Zwierlein et al., ibid. 92, 120403 (2004).

[4] A. Bulgac and Y. Yu, Phys. Rev. Lett. 88, 042504 (2002); 90, 222501 (2003); Int. J. Mod. Phys. E 13, 147 (2004).

[5] A. Bulgac and Y. Yu, Phys. Rev. Lett. 91, 190404 (2003); cond-mat/0406256.

[6] N. Nygaard, G. M. Bruun, C. W. Clark, and D. L. Feder, Phys. Rev. Lett. 90, 210402 (2003).

[7] C. A. R. Sá de Melo, M. Randeria, and J. R. Engelbrecht, Phys. Rev. Lett. 71, 3202 (1993).

[8] J. R. Engelbrecht, M. Randeria, and C. A. R. Sá de Melo,
Phys. Rev. B 55, 15153 (1997).

[9] H. Heiselberg, C. J. Pethick, H. Smith, and L. Viverit, Phys. Rev. Lett. 85, 2418 (2000); H. T. C. Stoof, M. Houbiers, C. A. Sackett, and R. G. Hulet, ibid. 76, 10 (1996).

[10] I. J. R. Aitchison, G. Metikas, and D. J. Lee, Phys. Rev. B 62, 6638 (2000).

[11] H. Kleinert, S. Schmidt, and A. Pelster, Ann. Phys. (to be published); Phys. Rev. Lett. 93, 160402 (2004).

[12] S. De Palo, C. Castellani, C. Di Castro, and B. K. Chakraverty, Phys. Rev. B 60, 564 (1999).

[13] P. M. Pizzochero, L. Viverit, and R. A. Broglia, Phys. Rev. Lett. 79, 3347 (1997); P. Donati and P. M. Pizzochero, ibid. 90, 211101 (2003); Nucl. Phys. A 742, 363 (2004).

[14] R. Epstein and G. Baym, Astrophys. J. 328, 680 (1988).

[15] Ø. Elgarøy and F. V. De Blasio, Astron. Astrophys. 370, 939 (2001). 